\title{
Benzoxazinoids in Prostate Cancer Patients after a Rye-Intensive Diet: Methods and Initial Results
}

Stine K. Steffensen ${ }^{a}$, Hans A. Pedersen ${ }^{a}$, Khem B. Adhikari ${ }^{a}$, Bente B. Laursen ${ }^{a}$, Claudia Jensen ${ }^{\mathrm{a}}$, Søren Høyer ${ }^{\mathrm{b}}$, Michael Borre ${ }^{\mathrm{c}}$, Helene H. Pedersen ${ }^{\mathrm{c}}$, Mette Borre $^{\mathrm{d}}$, David Edwards ${ }^{\mathrm{e}}$, Inge S. Fomsgaard ${ }^{\mathrm{a}}$.

${ }^{a}$ Department of Agroecology, Aarhus University, Forsøgsvej 1, DK-4200 Slagelse, Denmark.

${ }^{\mathrm{b}}$ Department of Pathology, Aarhus University Hospital, Nørrebrogade 44, DK-8000 Aarhus C, Denmark

${ }^{c}$ Department of Urology, Aarhus University Hospital, Palle Juul-Jensens Boulevard 99, DK-8200 Aarhus N, Denmark

${ }^{\mathrm{d}}$ Department of Medicine V (Hepatology and Gastroenterology), Aarhus University Hospital, Nørrebrogade 44, DK-8000 Aarhus C, Denmark

${ }^{\mathrm{e}}$ Department of Molecular Biology and Genetics, Aarhus University, Blichers Allé 20, DK-8830 Tjele, Denmark

*Corresponding author: stinek.steffensen@agro.au.dk

Short title: Benzoxazinoids in Prostate Cancer Patients after a Rye-Intensive Diet 


\section{Supplementary Online Tables}

Supplementary Table 1. Model parameters for the models used for investigations of the matrix effects.

\begin{tabular}{|c|c|c|c|c|c|c|c|c|c|c|c|c|}
\hline \multirow[t]{2}{*}{ Sample } & \multirow[t]{2}{*}{ Compound } & \multicolumn{10}{|c|}{ parameter } & \multirow[t]{2}{*}{ RMSPE } \\
\hline & & $a$ & $b$ & & $c$ & $p$ & & $q$ & & $r$ & & \\
\hline \multirow[t]{16}{*}{ Biopsy } & DIBOA-glc-hex & $-7.97 \times 10^{-4}$ & $1.00 \times 10^{0}$ & $* * *$ & $-8.69 \times 10^{-6}$ & $-2.06 \times 10^{-1}$ & $* * *$ & $4.74 \times 10^{-1}$ & $* * *$ & $3.61 \times 10^{-3}$ & & 0.056 \\
\hline & DIBOA-glc & $-5.60 \times 10^{-5}$ & $1.00 \times 10^{0}$ & $* * *$ & $8.54 \times 10^{-6}$ & $-2.82 \times 10^{-1}$ & $* * *$ & $4.96 \times 10^{-1}$ & $* * *$ & $4.03 \times 10^{-3}$ & & 0.062 \\
\hline & DIBOA & $-1.10 \times 10^{-1}$ & $1.20 \times 10^{0}$ & $* * *$ & $-3.64 \times 10^{-2}$ & $-1.60 \times 10^{-1}$ & & $2.45 \times 10^{-1}$ & & $7.58 \times 10^{-2}$ & & - \\
\hline & BOA & $1.13 \times 10^{-3}$ & $9.97 \times 10^{-1}$ & $* * *$ & $3.40 \times 10^{-4}$ & $-7.44 \times 10^{-2}$ & & $-1.54 \times 10^{-1}$ & & $3.15 \times 10^{-3}$ & & 0.093 \\
\hline & DIMBOA-glc & $-5.62 \times 10^{-3}$ & $1.01 \times 10^{0}$ & $* * *$ & $-1.43 \times 10^{-3}$ & $-2.47 \times 10^{-1}$ & $* * *$ & $4.54 \times 10^{-1}$ & $* * *$ & $7.90 \times 10^{-3}$ & $* *$ & 0.045 \\
\hline & DIMBOA & $-6.24 \times 10^{-2}$ & $1.11 \times 10^{0}$ & $* * *$ & $-1.57 \times 10^{-2}$ & $3.70 \times 10^{-5}$ & & $1.47 \times 10^{-2}$ & & $6.31 \times 10^{-2}$ & & - \\
\hline & HBOA-glc-hex & $-7.14 \times 10^{-4}$ & $1.00 \times 10^{0}$ & $* * *$ & $-2.89 \times 10^{-6}$ & $-1.14 \times 10^{-1}$ & $*$ & $3.34 \times 10^{-1}$ & $* * *$ & $1.09 \times 10^{-2}$ & $* * *$ & 0.052 \\
\hline & HBOA-glc & $-1.23 \times 10^{-3}$ & $1.00 \times 10^{0}$ & $* * *$ & $-1.44 \times 10^{-5}$ & $-1.76 \times 10^{-1}$ & $*$ & $3.68 \times 10^{-1}$ & $* *$ & $6.49 \times 10^{-3}$ & & 0.061 \\
\hline & HBOA & $1.63 \times 10^{-3}$ & $9.99 \times 10^{-1}$ & $* * *$ & $5.46 \times 10^{-6}$ & $-1.06 \times 10^{-1}$ & ، & $1.86 \times 10^{-1}$ & $*$ & $5.27 \times 10^{-3}$ & ، & 0.053 \\
\hline & HMBOA-glc & $-4.09 \times 10^{-4}$ & $1.00 \times 10^{0}$ & $* * *$ & $1.12 \times 10^{-5}$ & $-1.82 \times 10^{-1}$ & $*$ & $3.93 \times 10^{-1}$ & $* * *$ & $1.00 \times 10^{-2}$ & * & 0.081 \\
\hline & HMBOA & $3.67 \times 10^{-4}$ & $1.00 \times 10^{0}$ & $* * *$ & $9.41 \times 10^{-6}$ & $-9.40 \times 10^{-2}$ & & $1.19 \times 10^{-1}$ & & $4.41 \times 10^{-3}$ & & 0.069 \\
\hline & MBOA & $7.02 \times 10^{-4}$ & $1.00 \times 10^{0}$ & $* * *$ & $-8.56 \times 10^{-6}$ & $-6.13 \times 10^{-2}$ & & $-3.07 \times 10^{-1}$ & $* *$ & $1.13 \times 10^{-3}$ & & 0.083 \\
\hline & AAMPO & $8.08 \times 10^{-4}$ & $1.00 \times 10^{0}$ & $* * *$ & $7.65 \times 10^{-7}$ & $-1.31 \times 10^{-1}$ & & $2.86 \times 10^{-1}$ & $*$ & $6.04 \times 10^{-3}$ & & 0.095 \\
\hline & AAPO & $-3.55 \times 10^{-3}$ & $1.00 \times 10^{0}$ & $* * *$ & $-5.26 \times 10^{-5}$ & $-1.73 \times 10^{-1}$ & ، & $4.38 \times 10^{-1}$ & $* *$ & $6.52 \times 10^{-3}$ & & 0.101 \\
\hline & AMPO & $-2.15 \times 10^{-2}$ & $1.02 \times 10^{0}$ & $* * *$ & $1.33 \times 10^{-4}$ & $-6.19 \times 10^{-1}$ & $*$ & $1.32 \times 10^{0}$ & $* *$ & $4.59 \times 10^{-2}$ & $* *$ & 0.144 \\
\hline & APO & $-1.53 \times 10^{-2}$ & $1.02 \times 10^{0}$ & $* * *$ & $-2.84 \times 10^{-4}$ & $-4.93 \times 10^{-1}$ & $*$ & $1.05 \times 10^{0}$ & $* *$ & $2.45 \times 10^{-2}$ & ، & 0.141 \\
\hline \multirow[t]{16}{*}{ Plasma } & DIBOA-glc-hex & $1.86 \times 10^{-3}$ & $9.99 \times 10^{-1}$ & $* * *$ & $3.65 \times 10^{-4}$ & $-5.12 \times 10^{-1}$ & $* * *$ & $5.97 \times 10^{-1}$ & $* * *$ & $7.22 \times 10^{-3}$ & & 0.048 \\
\hline & DIBOA-glc & $-1.41 \times 10^{-3}$ & $1.00 \times 10^{0}$ & $* * *$ & $-2.10 \times 10^{-5}$ & $-4.89 \times 10^{-1}$ & $* * *$ & $5.68 \times 10^{-1}$ & $* * *$ & $1.67 \times 10^{-2}$ & $* *$ & 0.049 \\
\hline & DIBOA & $7.04 \times 10^{-4}$ & $9.99 \times 10^{-1}$ & $* * *$ & $5.93 \times 10^{-6}$ & $-1.89 \times 10^{-1}$ & & $3.55 \times 10^{-2}$ & & $1.01 \times 10^{-1}$ & $*$ & - \\
\hline & BOA & $1.70 \times 10^{-4}$ & $1.00 \times 10^{0}$ & $* * *$ & $-6.40 \times 10^{-6}$ & $-4.49 \times 10^{-1}$ & $* * *$ & $4.90 \times 10^{-1}$ & $* * *$ & $1.25 \times 10^{-2}$ & * & 0.058 \\
\hline & DIMBOA-glc & $-2.72 \times 10^{-4}$ & $1.00 \times 10^{0}$ & $* * *$ & $2.64 \times 10^{-4}$ & $-4.77 \times 10^{-1}$ & $* * *$ & $5.27 \times 10^{-1}$ & $* *$ & $1.47 \times 10^{-2}$ & * & 0.043 \\
\hline & DIMBOA & $9.26 \times 10^{-4}$ & $1.00 \times 10^{0}$ & $* * *$ & $1.48 \times 10^{-4}$ & $-2.38 \times 10^{-1}$ & & $4.56 \times 10^{-2}$ & & $5.95 \times 10^{-2}$ & & - \\
\hline & HBOA-glc-hex & $-6.04 \times 10^{-4}$ & $1.00 \times 10^{0}$ & $* * *$ & $-1.30 \times 10^{-5}$ & $-5.26 \times 10^{-1}$ & $* * *$ & $6.70 \times 10^{-1}$ & $* * *$ & $1.58 \times 10^{-2}$ & * & 0.058 \\
\hline & HBOA-glc & $-1.92 \times 10^{-3}$ & $1.00 \times 10^{0}$ & $* * *$ & $-1.35 \times 10^{-5}$ & $-4.92 \times 10^{-1}$ & $* * *$ & $5.90 \times 10^{-1}$ & $* * *$ & $2.01 \times 10^{-2}$ & $* *$ & 0.068 \\
\hline & HBOA & $6.32 \times 10^{-5}$ & $1.00 \times 10^{0}$ & $* * *$ & $-4.10 \times 10^{-6}$ & $-4.93 \times 10^{-1}$ & $* * *$ & $5.67 \times 10^{-1}$ & $* * *$ & $1.27 \times 10^{-2}$ & $*$ & 0.054 \\
\hline & HMBOA-glc & $-2.31 \times 10^{-3}$ & $1.00 \times 10^{0}$ & $* * *$ & $-2.01 \times 10^{-5}$ & $-5.42 \times 10^{-1}$ & $* * *$ & $6.59 \times 10^{-1}$ & $* * *$ & $1.66 \times 10^{-2}$ & * & 0.056 \\
\hline & HMBOA & $-2.04 \times 10^{-4}$ & $1.00 \times 10^{0}$ & $* * *$ & $-1.19 \times 10^{-6}$ & $-4.49 \times 10^{-1}$ & $* * *$ & $4.92 \times 10^{-1}$ & $* *$ & $1.38 \times 10^{-2}$ & * & 0.057 \\
\hline & MBOA & $2.45 \times 10^{-7}$ & $1.00 \times 10^{0}$ & $* * *$ & $4.75 \times 10^{-6}$ & $-4.23 \times 10^{-1}$ & $* * *$ & $4.29 \times 10^{-1}$ & & $9.01 \times 10^{-3}$ & ، & 0.045 \\
\hline & AAMPO & $7.83 \times 10^{-3}$ & $9.87 \times 10^{-1}$ & $* * *$ & $1.68 \times 10^{-3}$ & $-4.15 \times 10^{-1}$ & $* * *$ & $5.10 \times 10^{-1}$ & $* *$ & $-6.66 \times 10^{-3}$ & & 0.071 \\
\hline & AAPO & $1.76 \times 10^{-3}$ & $9.99 \times 10^{-1}$ & $* * *$ & $2.37 \times 10^{-5}$ & $-4.12 \times 10^{-1}$ & $* * *$ & $5.61 \times 10^{-1}$ & $* * *$ & $3.42 \times 10^{-3}$ & & 0.079 \\
\hline & AMPO & $9.37 \times 10^{-3}$ & $9.81 \times 10^{-1}$ & $* *$ & $7.94 \times 10^{-5}$ & $-8.83 \times 10^{-1}$ & $* *$ & $1.68 \times 10^{0}$ & $* * *$ & $8.57 \times 10^{-3}$ & & 0.151 \\
\hline & APO & $7.01 \times 10^{-3}$ & $9.86 \times 10^{-1}$ & $* * *$ & $2.17 \times 10^{-3}$ & $-5.92 \times 10^{-1}$ & $* *$ & $1.00 \times 10^{0}$ & $* * *$ & $8.93 \times 10^{-3}$ & & 0.125 \\
\hline \multirow[t]{4}{*}{ Urine } & DIBOA-glc-hex & $-6.29 \times 10^{-6}$ & $1.00 \times 10^{0}$ & $* * *$ & $1.21 \times 10^{-3}$ & $-3.94 \times 10^{-5}$ & & $-2.80 \times 10^{-2}$ & & $1.21 \times 10^{-1}$ & & 1.373 \\
\hline & DIBOA-glc & $8.63 \times 10^{-6}$ & $1.00 \times 10^{0}$ & $* * *$ & $1.09 \times 10^{-3}$ & $-3.53 \times 10^{-4}$ & & $-7.92 \times 10^{-2}$ & ، & $1.74 \times 10^{-1}$ & & 2.403 \\
\hline & DIBOA & $-6.10 \times 10^{-5}$ & $1.00 \times 10^{0}$ & $* * *$ & $-2.32 \times 10^{-3}$ & $-3.87 \times 10^{-4}$ & & $-6.73 \times 10^{-2}$ & & $1.54 \times 10^{-2}$ & & 3.875 \\
\hline & BOA & $-1.95 \times 10^{-5}$ & $1.00 \times 10^{0}$ & $* * *$ & $-5.95 \times 10^{-4}$ & $3.29 \times 10^{-5}$ & & $-2.52 \times 10^{-1}$ & $* *$ & $6.00 \times 10^{-2}$ & & 4.242 \\
\hline
\end{tabular}




\begin{tabular}{|c|c|c|c|c|c|c|c|c|c|c|}
\hline DIMBOA-glc & $-8.24 \times 10^{-6}$ & $1.00 \times 10^{0}$ & $* * *$ & $7.42 \times 10^{-4}$ & $5.56 \times 10^{-5}$ & $-7.37 \times 10^{-2}$ & & $5.35 \times 10^{-2}$ & & 3.500 \\
\hline DIMBOA & $1.99 \times 10^{-5}$ & $9.99 \times 10^{-1}$ & $* * *$ & $1.58 \times 10^{-3}$ & $1.82 \times 10^{-4}$ & $-2.37 \times 10^{-1}$ & $*$ & $7.56 \times 10^{-2}$ & & 5.116 \\
\hline HBOA-glc-hex & $-3.36 \times 10^{-5}$ & $1.00 \times 10^{0}$ & $* * *$ & $7.86 \times 10^{-4}$ & $1.87 \times 10^{-4}$ & $-5.40 \times 10^{-2}$ & & $1.49 \times 10^{-1}$ & & 1.974 \\
\hline HBOA-glc & $-4.87 \times 10^{-7}$ & $9.99 \times 10^{-1}$ & $* * *$ & $2.69 \times 10^{-4}$ & $-1.04 \times 10^{-4}$ & $-1.02 \times 10^{-1}$ & & $1.34 \times 10^{0}$ & $* *$ & 3.487 \\
\hline HBOA & $-1.62 \times 10^{-6}$ & $1.00 \times 10^{0}$ & $* * *$ & $-1.76 \times 10^{-4}$ & $2.11 \times 10^{-4}$ & $-1.66 \times 10^{-1}$ & $* *$ & $8.97 \times 10^{-2}$ & & 3.012 \\
\hline HMBOA-glc & $-5.45 \times 10^{-6}$ & $1.00 \times 10^{0}$ & $* * *$ & $-6.85 \times 10^{-4}$ & $-1.98 \times 10^{-4}$ & $-1.08 \times 10^{-1}$ & ‘ & $8.21 \times 10^{-2}$ & & 3.159 \\
\hline HMBOA & $-4.40 \times 10^{-6}$ & $9.99 \times 10^{-1}$ & $* * *$ & $1.78 \times 10^{-4}$ & $3.61 \times 10^{-4}$ & $-2.67 \times 10^{-1}$ & $* *$ & $1.37 \times 10^{-1}$ & & 4.555 \\
\hline MBOA & $5.06 \times 10^{-6}$ & $1.00 \times 10^{0}$ & $* * *$ & $-4.96 \times 10^{-4}$ & $-7.35 \times 10^{-5}$ & $-1.80 \times 10^{-1}$ & $* * *$ & $5.27 \times 10^{-2}$ & & 2.584 \\
\hline AAMPO & $-6.16 \times 10^{-6}$ & $1.00 \times 10^{0}$ & $* * *$ & $-2.25 \times 10^{-3}$ & $3.70 \times 10^{-4}$ & $-1.76 \times 10^{-2}$ & & $1.44 \times 10^{-1}$ & & 0.737 \\
\hline AAPO & $-3.43 \times 10^{-5}$ & $1.00 \times 10^{0}$ & $* * *$ & $-4.75 \times 10^{-4}$ & $9.12 \times 10^{-5}$ & $-4.26 \times 10^{-2}$ & & $1.31 \times 10^{-1}$ & & 3.177 \\
\hline AMPO & $-3.78 \times 10^{-6}$ & $9.99 \times 10^{-1}$ & $* * *$ & $1.06 \times 10^{-3}$ & $-8.04 \times 10^{-4}$ & $7.55 \times 10^{-2}$ & & $2.16 \times 10^{-1}$ & ‘ & 1.18 \\
\hline APO & $-3.12 \times 10^{-5}$ & $1.00 \times 10^{0}$ & $* * *$ & $-1.56 \times 10^{-3}$ & $1.39 \times 10^{-4}$ & $-5.48 \times 10^{-3}$ & & $1.30 \times 10^{-1}$ & & 2.269 \\
\hline
\end{tabular}

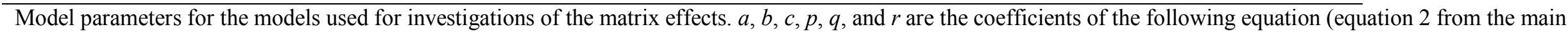
text):

$$
\text { conc }_{\text {quant }}=(a+p \times m) \times \text { conc }_{\text {actual }}{ }^{2}+(b+q \times m) \times \text { conc }_{\text {actual }}+(c+r \times m)
$$

$m=1$ or 0 depending on the presence or absence of sample matrix.

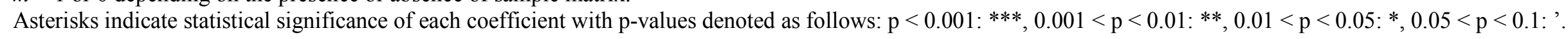


Supplementary Table 2. Mean concentrations of the triplicate standard curves in solvent and in sample matrix extract for the three different sample matrices.

\begin{tabular}{|c|c|c|c|c|c|c|c|c|c|c|c|c|c|c|c|c|c|c|}
\hline \multirow[b]{2}{*}{\begin{tabular}{|l|} 
Compound \\
\end{tabular}} & \multicolumn{6}{|c|}{ Biopsy } & \multicolumn{6}{|c|}{ Plasma } & \multicolumn{6}{|c|}{ Urine } \\
\hline & $\begin{array}{c}\text { Nominal } \\
\text { Concentrat } \\
\text { ion } \\
(\mathrm{ng} / \mathrm{mL})\end{array}$ & $\begin{array}{c}\text { Mean } \\
\text { solvent } \\
\text { concentrati } \\
\text { on }\end{array}$ & $\begin{array}{l}\text { CV\% in } \\
\text { solvent }\end{array}$ & $\begin{array}{c}\text { Mean } \\
\text { matrix } \\
\text { concentrati } \\
\text { on }\end{array}$ & $\begin{array}{l}\mathrm{CV} \% \text { in } \\
\text { sample } \\
\text { matrix }\end{array}$ & ME\% & \begin{tabular}{|c|} 
Nominal \\
Concentrat \\
ion \\
$(\mathrm{ng} / \mathrm{mL})$
\end{tabular} & \begin{tabular}{|c|} 
Mean \\
solvent \\
concentrati \\
on
\end{tabular} & $\begin{array}{l}\text { CV\% in } \\
\text { solvent }\end{array}$ & $\begin{array}{c}\text { Mean } \\
\text { matrix } \\
\text { concentrati } \\
\text { on }\end{array}$ & $\begin{array}{l}\mathrm{CV} \% \text { in } \\
\text { sample } \\
\text { matrix }\end{array}$ & ME\% & \begin{tabular}{|c|} 
Nominal \\
Concentrat \\
ion \\
$(\mathrm{ng} / \mathrm{mL})$ \\
\end{tabular} & \begin{tabular}{|c|} 
Mean \\
solvent \\
concentrati \\
on
\end{tabular} & $\begin{array}{l}\text { CV\% in } \\
\text { solvent }\end{array}$ & \begin{tabular}{c|} 
Mean \\
matrix \\
concentrati \\
on
\end{tabular} & $\begin{array}{l}\mathrm{CV} \% \text { in } \\
\text { sample } \\
\text { matrix }\end{array}$ & ME\% \\
\hline \multirow[t]{6}{*}{ HBOA } & 0 & & & \begin{tabular}{|l|l}
0.00130 \\
\end{tabular} & 90.7 & & 0 & & & 0 & 0 & & 0 & & & \begin{tabular}{l|}
0.0412 \\
\end{tabular} & 39.6 & \\
\hline & 0.00625 & 0.00558 & 68.9 & \begin{tabular}{l|l}
0.0118 \\
\end{tabular} & 37.6 & 211 & 0.00625 & 0.00542 & 47.8 & \begin{tabular}{|l|l|}
0.0173 \\
\end{tabular} & 10.2 & 319 & 0.391 & 0.401 & 12.1 & 0.407 & 4.77 & 101 \\
\hline & 0.0250 & 0.0279 & 11.5 & 0.0380 & 6.08 & 136 & 0.0250 & 0.0268 & 17.3 & 0.0668 & 3.68 & 249 & 1.56 & 1.53 & 11.3 & 1.43 & 8.78 & 93.9 \\
\hline & 0.100 & 0.0997 & 8.46 & 0.126 & 9.52 & 126 & 0.100 & 0.109 & 4.20 & 0.200 & 18.2 & 183 & 6.25 & 6.18 & 6.18 & 5.27 & 10.7 & 85.4 \\
\hline & 0.400 & 0.397 & 2.17 & 0.456 & 0.13 & 115 & 0.400 & 0.388 & 11.1 & 0.503 & 15.6 & 130 & 25.0 & 25.1 & 2.04 & 21.1 & 7.36 & 84.0 \\
\hline & $\frac{1.60}{1.60}$ & 1.60 & 6.31 & $\begin{array}{l}.70 \\
1.64\end{array}$ & 8.25 & 102 & $\begin{array}{l}.900 \\
1.60\end{array}$ & $\frac{0.000}{1.60}$ & 4.42 & \begin{tabular}{l|}
1.27 \\
\end{tabular} & 4.17 & 79.2 & 100 & 100.0 & 1.73 & 85.7 & 8.60 & 85.7 \\
\hline \multirow[t]{6}{*}{ BOA } & 0 & & & 0.00 & - & & 0 & - & - & \begin{tabular}{|l|}
0.00101 \\
\end{tabular} & 141 & & 0 & - & 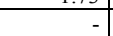 & \begin{tabular}{l|}
0.0245 \\
\end{tabular} & 45.3 & 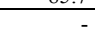 \\
\hline & 0.00625 & 0.00785 & 93.3 & \begin{tabular}{l|l|}
0.00754 \\
\end{tabular} & 1.22 & 96.1 & \begin{tabular}{l|l|}
0.00625 \\
\end{tabular} & 0.00548 & 36.0 & \begin{tabular}{|l|l|}
0.0173 \\
\end{tabular} & 31.9 & 315 & \begin{tabular}{l|l|}
0.391 \\
\end{tabular} & 0.399 & 8.60 & 0.342 & 16.8 & 85.7 \\
\hline & 0.0250 & 0.0199 & 5.25 & \begin{tabular}{l|l|}
0.0290 \\
\end{tabular} & 7.61 & 146 & \begin{tabular}{l|}
0.0250 \\
\end{tabular} & 0.0257 & 20.6 & \begin{tabular}{l|l|}
0.0627 \\
\end{tabular} & 10.2 & 244 & 1.56 & 1.53 & 10.6 & 1.27 & 15.8 & 83.0 \\
\hline & 0.100 & 0.0997 & 12.8 & 0.0908 & 15.0 & 91.1 & 0.100 & 0.113 & 6.01 & 0.187 & 14.2 & 165 & 6.25 & 6.19 & 4.78 & 4.71 & 19.8 & 76.0 \\
\hline & 0.400 & 0.405 & 15.9 & 0.320 & 12.2 & 79.0 & 0.400 & 0.383 & 10.1 & 0.488 & 15.1 & 127 & 25.0 & 25.10 & 3.16 & 18.8 & 17.3 & 74.8 \\
\hline & 1.60 & 1.597 & 6.30 & 1.17 & 16.2 & 73.1 & 1.60 & 1.60 & 4.72 & 1.26 & 6.67 & 78.4 & 100 & 99.8 & 1.91 & 75.0 & 13.6 & 75.2 \\
\hline \multirow[t]{6}{*}{ MBOA } & 0 & & & 0.00 & - & & 0 & & & 0 & 0 & & 0 & & & \begin{tabular}{l|}
0.0248 \\
\end{tabular} & 3.14 & \\
\hline & 0.00625 & 0.00559 & 36.9 & 0.00428 & 26.7 & 76.6 & 0.00625 & 0.00550 & 39.2 & \begin{tabular}{|l|}
0.0132 \\
\end{tabular} & 18.4 & 240 & 0.391 & 0.399 & 10.8 & 0.373 & 9.07 & 93.5 \\
\hline & 0.0250 & 0.0267 & 1.35 & $\begin{array}{r}0.0219 \\
\end{array}$ & 13.8 & 82.0 & \begin{tabular}{|r|}
0.0250 \\
\end{tabular} & 0.0262 & 18.7 & \begin{tabular}{l|l}
0.0584 \\
\end{tabular} & 8.24 & 223 & \begin{tabular}{|c|}
.56 \\
\end{tabular} & 1.55 & 10.3 & \begin{tabular}{|l|}
1.35 \\
\end{tabular} & 7.18 & 87.5 \\
\hline & 0.100 & 0.106 & 22.2 & \begin{tabular}{|c|}
0.0774 \\
\end{tabular} & 9.89 & 73.3 & \begin{tabular}{|c|}
0.0200 \\
0.100
\end{tabular} & 0.111 & 8.70 & \begin{tabular}{|c|}
0.180 \\
\end{tabular} & 13.9 & 163 & 6.25 & 6.11 & 6.06 & 5.05 & 12.1 & 82.7 \\
\hline & 0.400 & 0.392 & 7.41 & 0.256 & 21.2 & 65.4 & 0.400 & 0.386 & 11.1 & 0.460 & 10.9 & 119 & 25.0 & 25.20 & 2.10 & 20.7 & 8.98 & 82.0 \\
\hline & 1.60 & 1.60 & 7.49 & \begin{tabular}{l|l|}
0.957 \\
\end{tabular} & 21.4 & 59.7 & 1.60 & 1.60 & 4.42 & 1.22 & 2.50 & 76.3 & 100 & 100 & 1.90 & 81.4 & 7.43 & 81.4 \\
\hline \multirow[t]{6}{*}{ HMBOA } & 0 & & & 0.00116 & 173 & & 0 & - & - & 0 & 0 & & 0 & & & \begin{tabular}{l|l|}
0.102 \\
\end{tabular} & 93.0 & \\
\hline & 0.00625 & 0.00636 & 61.9 & \begin{tabular}{|c|}
0.0102 \\
\end{tabular} & 45.9 & 160 & 0.00625 & 0.00583 & 39.5 & \begin{tabular}{l|}
0.0177 \\
\end{tabular} & 7.05 & 304 & \begin{tabular}{|l|}
0.391 \\
\end{tabular} & 0.406 & 9.30 & $\begin{array}{l}.1108 \\
0.408\end{array}$ & 17.1 & 100 \\
\hline & 0.0250 & 0.0248 & 2.98 & \begin{tabular}{l|l}
0.0366 \\
\end{tabular} & 8.74 & 148 & 0.0250 & 0.0238 & 18.1 & \begin{tabular}{|l|}
0.0673 \\
\end{tabular} & 14.6 & 283 & 1.56 & 1.51 & 13.1 & 1.32 & 11.8 & 87.4 \\
\hline & 0.100 & 0.0990 & 18.5 & 0.120 & 4.10 & 122 & 0.100 & 0.116 & 4.92 & \begin{tabular}{l|}
0.192 \\
\end{tabular} & 12.1 & 166 & 6.25 & 6.12 & 5.63 & 4.82 & 16.8 & 78.8 \\
\hline & 0.400 & 0.401 & 1.44 & \begin{tabular}{l|l|}
0.427 \\
\end{tabular} & 8.23 & 106 & 0.400 & 0.383 & 9.61 & \begin{tabular}{l|l|}
0.482 \\
\end{tabular} & 14.6 & 126 & 25.0 & 25.17 & 2.65 & \begin{tabular}{|l|}
18.5 \\
\end{tabular} & 13.6 & 73.5 \\
\hline & 1.60 & 1.60 & 7.58 & \begin{tabular}{l|}
1.56 \\
\end{tabular} & 10.4 & 97.3 & $\begin{array}{l}1.60 \\
\end{array}$ & 1.60 & 5.30 & 1.26 & 5.15 & 78.8 & 100 & 99.8 & 0.29 & 76.9 & 14.9 & 77.1 \\
\hline \multirow[t]{6}{*}{ DIBOA } & 0 & & & 0 & 0 & - & 0 & - & - & 0 & 0 & - & 0 & - & & 0 & 0 & \\
\hline & 0.00625 & 0 & 0 & 0 & 0 & 0 & 0.00625 & 0 & 0 & 0 & 0 & 0 & \begin{tabular}{|c|}
0.391 \\
\end{tabular} & 0.356 & \begin{tabular}{|l|}
13.9 \\
\end{tabular} & 0.401 & 15.1 & 113 \\
\hline & 0.0250 & 0 & 0 & 0 & 0 & 0 & 0.0250 & 0 & 0 & 0 & 0 & 0 & 1.56 & 1.63 & 2.48 & 1.39 & 12.6 & 85.5 \\
\hline & $\frac{0.0200}{0.100}$ & 0.123 & 14.9 & \begin{tabular}{|l|}
0.181 \\
\end{tabular} & 5.76 & 147 & \begin{tabular}{r|}
0.0230 \\
0.100
\end{tabular} & 0.100 & \begin{tabular}{|l|}
19.9 \\
\end{tabular} & \begin{tabular}{|r|}
0.203 \\
\end{tabular} & 24.1 & 203 & $\frac{1.00}{6.25}$ & 6.05 & 6.46 & $\begin{array}{l}5.37 \\
5.77\end{array}$ & $\frac{2.0}{22.5}$ & 86.6 \\
\hline & 0.400 & 0.424 & 18.8 & \begin{tabular}{l|l|}
0.572 \\
\end{tabular} & 6.93 & 135 & 0.400 & 0.400 & 12.1 & \begin{tabular}{l|}
0.485 \\
\end{tabular} & 14.4 & 121 & 25.0 & 24.5 & 3.27 & 23.3 & 15.1 & 95.1 \\
\hline & 1.60 & 1.59 & 5.34 & \begin{tabular}{l|}
1.65 \\
\end{tabular} & 4.96 & 104 & $\begin{array}{l}1.60 \\
\end{array}$ & 1.60 & 3.90 & $\begin{array}{l}1.27 \\
\end{array}$ & 4.33 & 79.6 & 100 & 99.8 & 7.88 & 89.1 & 5.41 & 89.2 \\
\hline \multirow[t]{6}{*}{ DIMBOA } & 0 & - & - & 0 & 0 & & 0 & & - & 0 & 0 & & 0 & & & 0 & 0 & \\
\hline & 0.00625 & 0 & 0 & 0 & 0 & 0 & 0.00625 & 0 & 0 & 0 & 0 & 0 & 0.391 & 0.438 & 24.5 & 0.391 & 12.9 & 89.3 \\
\hline & 0.0250 & 0 & 0 & 0 & 0 & 0 & \begin{tabular}{|c|}
0.0250 \\
\end{tabular} & 0 & 0 & 0.115 & 0 & 0 & 1.56 & 1.43 & 10.7 & 1.24 & 6.30 & 86.5 \\
\hline & 0.100 & 0.0944 & 25.6 & 0.159 & 34.0 & 168 & 0.100 & 0.100 & 37.3 & 0.162 & 25.7 & 162 & 6.25 & 5.86 & 11.7 & 4.62 & 14.6 & 78.7 \\
\hline & 0.400 & 0.417 & 22.0 & 0.486 & 6.48 & 117 & 0.400 & 0.400 & 12.1 & 0.439 & 15.4 & 110 & 25.0 & 25.6 & 2.26 & 19.6 & 13.6 & 76.5 \\
\hline & 1.60 & 1.60 & 4.40 & 1.68 & 8.43 & 105 & 1.60 & 1.60 & 13.5 & 1.12 & 7.78 & 70.2 & 100 & 100.0 & 2.70 & 78.3 & 16.1 & 78.3 \\
\hline \multirow[t]{6}{*}{ HBOA-glc } & & & & 0.00584 & 118 & & 0 & & & \begin{tabular}{l|l|}
0.00663 \\
\end{tabular} & 106 & & 0 & & & 0.560 & 223 & \\
\hline & 0.00625 & 0.00494 & 53.7 & \begin{tabular}{|c|}
0.0137 \\
\end{tabular} & 94.6 & 277 & \begin{tabular}{l|l|}
0.00625 \\
\end{tabular} & 0.00593 & 37.8 & \begin{tabular}{|c|}
0.0242 \\
\end{tabular} & 18.8 & 409 & \begin{tabular}{l|l|}
0.391 \\
\end{tabular} & 0.394 & 12.3 & 1.68 & 76.4 & 425 \\
\hline & 0.0250 & 0.0296 & 6.59 & \begin{tabular}{l|}
0.0444 \\
\end{tabular} & 19.2 & 150 & \begin{tabular}{l|}
0.0250 \\
\end{tabular} & 0.0240 & 33.5 & \begin{tabular}{|l|l|}
0.0775 \\
\end{tabular} & 3.6 & 324 & 1.56 & 1.52 & 12.4 & 2.80 & 48.4 & 184 \\
\hline & 0.100 & 0.105 & 14.5 & 0.151 & 16.5 & 144 & 0.100 & 0.113 & 5.91 & 0.208 & 16.9 & 184 & 6.25 & 6.34 & 6.61 & 6.82 & 21.9 & 107 \\
\hline & 0.400 & 0.390 & 5.90 & 0.510 & 5.45 & 131 & 0.400 & 0.386 & 11.4 & 0.520 & 16.3 & 135 & 25.0 & 24.9 & 2.90 & 23.8 & 12.0 & 95.4 \\
\hline & 1.60 & 1.60 & 6.16 & 1.75 & 7.82 & 109 & 1.60 & 1.60 & 6.25 & 1.31 & 6.48 & 82.1 & 100 & 99.9 & 2.05 & 90.0 & 8.76 & 90.0 \\
\hline \multirow[t]{6}{*}{ HBOA-glc-hex } & 0 & & & 0.000774 & 140 & & 0 & & & 0 & 0 & & 0 & & 4 & \begin{tabular}{l|l|}
0.0489 \\
\end{tabular} & 124 & \\
\hline & 0.00625 & 0.00574 & 21.7 & \begin{tabular}{|l|}
0.0183 \\
\end{tabular} & 18.4 & 319 & 0.00625 & 0.00531 & 74.9 & \begin{tabular}{|l|}
0.0197 \\
\end{tabular} & 16.6 & 371 & 0.391 & 0.406 & 2.35 & 0.508 & 17.3 & 125 \\
\hline & 0.0250 & 0.0262 & 6.42 & 0.0473 & 5.52 & 181 & 0.0250 & 0.0253 & 15.0 & \begin{tabular}{l|l|}
0.0775 \\
\end{tabular} & 8.41 & 307 & 1.56 & 1.50 & 9.82 & 1.68 & 7.04 & 112 \\
\hline & 0.100 & $\begin{array}{r}0.105 \\
0.105 \\
\end{array}$ & 8.51 & \begin{tabular}{|c|}
0.149 \\
\end{tabular} & 12.1 & 142 & o. 0.100 & $\begin{array}{c}0.119 \\
0.119\end{array}$ & 11.9 & 0.216 & $\begin{array}{l}0.71 \\
16.5 \\
\end{array}$ & 181 & 6.25 & 6.23 & 4.46 & 5.99 & 6.38 & 96.1 \\
\hline & 0.400 & 0.393 & 6.77 & 0.516 & 8.31 & 131 & 0.400 & 0.377 & 11.2 & 0.533 & 16.3 & 142 & 25.0 & 25.1 & 1.66 & 23.9 & 6.70 & 95.5 \\
\hline & 1.60 & 1.60 & 7.04 & \begin{tabular}{l|l|}
1.85 \\
\end{tabular} & 2.97 & 116 & \begin{tabular}{l|l}
1.60 \\
\end{tabular} & 1.60 & 3.81 & 1.35 & 4.33 & 84.4 & 100 & 99.7 & 0.579 & 96.3 & 4.95 & 96.6 \\
\hline \multirow[t]{4}{*}{ HMBOA-glc } & & & & \begin{tabular}{l|l}
0.00936 \\
\end{tabular} & 91.1 & & 0 & & & 0 & 0 & & 0 & & & \begin{tabular}{l|l|}
0.0215 \\
\end{tabular} & 103 & \\
\hline & 0.00625 & 0.00544 & 82.3 & \begin{tabular}{|l|}
0.0171 \\
\end{tabular} & 10.5 & 314 & 0.00625 & 0.00611 & 42.1 & 0.0202 & 31.5 & 330 & 0.391 & 0.399 & 9.78 & 0.426 & 10.0 & 107 \\
\hline & 0.0250 & 0.0284 & 4.07 & (0.0521 & 9.32 & 184 & \begin{tabular}{|c|}
0.0250 \\
\end{tabular} & 0.0230 & 6.65 & \begin{tabular}{l|l|}
0.0798 \\
\end{tabular} & 6.12 & 346 & 1.56 & 1.52 & 12.3 & 1.51 & 11.8 & 99.1 \\
\hline & 0.100 & 0.101 & 8.04 & 0.146 & 11.7 & 145 & 0.100 & 0.114 & 5.72 & 0.212 & 13.5 & 187 & 6.25 & 6.23 & 7.21 & 5.53 & 12.9 & 88.8 \\
\hline
\end{tabular}




\begin{tabular}{|c|c|c|c|c|c|c|c|c|c|c|c|c|c|c|c|c|c|c|}
\hline & 0.400 & 0.396 & 9.16 & 0.533 & 2.67 & 135 & \begin{tabular}{l|l|}
0.400 \\
\end{tabular} & 0.386 & 7.68 & $\begin{array}{ll}0.530 \\
\end{array}$ & \begin{tabular}{|l|}
15.8 \\
\end{tabular} & 137 & 25.0 & 25.1 & 3.25 & 22.4 & \begin{tabular}{ll|}
12.3 \\
\end{tabular} & 89.2 \\
\hline & 1.60 & 1.60 & 8.75 & 1.77 & 9.97 & 111 & 1.60 & 1.60 & 4.38 & 1.29 & 4.66 & 80.8 & 100 & 100.0 & 1.05 & 87.3 & 8.60 & 87.3 \\
\hline \multirow{6}{*}{ DIBOA-glc } & 0 & & & 0.00149 & 173 & & 0 & & - & 0 & 0 & & 0 & - & & \begin{tabular}{|l|l|}
0.147 \\
\end{tabular} & $\begin{array}{l}47.6 \\
\end{array}$ & \\
\hline & 0.00625 & 0.00694 & 62.0 & 0.0132 & 45.2 & 190 & 0.00625 & 0.00498 & 93.6 & $\begin{array}{l}0.0213 \\
\end{array}$ & \begin{tabular}{ll|}
14.4 \\
\end{tabular} & 429 & \begin{tabular}{|l|}
0.391 \\
\end{tabular} & $\begin{array}{l}0.387 \\
\end{array}$ & 10.6 & \begin{tabular}{l|l|}
0.521 \\
\end{tabular} & 22.2 & 135 \\
\hline & 0.0250 & 0.0217 & 13.4 & 0.0421 & 13.6 & 194 & 0.0250 & 0.0279 & 26.5 & $\begin{array}{l}0.0699 \\
\end{array}$ & 16.3 & 250 & 1.56 & 1.56 & 10.9 & 1.65 & \begin{tabular}{|l|}
9.87 \\
\end{tabular} & 105 \\
\hline & 0.100 & 0.102 & 1.13 & 0.151 & 3.64 & 148 & 0.100 & 0.113 & 1.35 & 0.208 & 8.48 & 183 & 6.25 & 6.34 & 4.82 & 6.01 & \begin{tabular}{|l|}
4.95 \\
\end{tabular} & 94.8 \\
\hline & 0.400 & 0.401 & 2.60 & 0.556 & 6.71 & 139 & 0.400 & 0.382 & 8.39 & 0.506 & 13.5 & 132 & 25.0 & 24.9 & 2.63 & 22.8 & \begin{tabular}{|c|}
9.87 \\
\end{tabular} & 91.7 \\
\hline & 1.60 & 1.60 & 1.65 & 1.68 & 8.36 & 105 & 1.60 & 1.60 & 3.20 & 1.29 & 4.49 & 80.2 & 100 & 100.1 & 4.41 & 88.8 & \begin{tabular}{|l|}
4.13 \\
\end{tabular} & 88.7 \\
\hline \multirow{6}{*}{ DIBOA-glc-hex } & 0 & - & & 0.00 & 0 & & 0 & - & - & 0 & 0 & & 0 & - & - & 0.00922 & 479 & \\
\hline & 0.00625 & 0.00600 & 47.9 & 0.0111 & 13.8 & 185 & \begin{tabular}{|c|}
0.00625 \\
\end{tabular} & 0.00588 & 108 & 0.0110 & 28.7 & 188 & 0.391 & 0.403 & 12.8 & 0.497 & 13.5 & 123 \\
\hline & 0.0250 & 0.0248 & 15.8 & 0.0473 & 11.0 & 190 & \begin{tabular}{l|}
0.0250 \\
\end{tabular} & 0.0257 & 14.2 & 0.0672 & 6.40 & 262 & 1.56 & 1.51 & 11.5 & 1.67 & 7.2 & 111 \\
\hline & 0.100 & 0.106 & 3.56 & 0.150 & 19.0 & 141 & \begin{tabular}{l|l|}
0.100 \\
\end{tabular} & 0.115 & 9.65 & 0.202 & 14.8 & 176 & 6.25 & 6.23 & 5.24 & 6.10 & \begin{tabular}{|l|}
3.00 \\
\end{tabular} & 98.0 \\
\hline & 0.400 & 0.393 & 3.87 & 0.552 & 4.57 & 141 & 0.400 & 0.383 & 11.1 & 0.497 & 12.1 & 130 & 25.0 & 25.1 & 2.01 & 24.5 & \begin{tabular}{|l|}
5.67 \\
\end{tabular} & 97.6 \\
\hline & 1.60 & 1.60 & 5.56 & 1.84 & 4.94 & 115 & 1.60 & 1.61 & 3.43 & 1.27 & 3.43 & 79.0 & 100 & 99.9 & 1.15 & 96.8 & $\begin{array}{l}3.09 \\
\end{array}$ & 96.9 \\
\hline \multirow[t]{6}{*}{ DIMBOA-glc } & 0 & & & 0.00 & 0 & & 0 & & -- & 0 & 0 & & 0 & & & 0 & 0 & \\
\hline & 0.00625 & 0.00327 & 173 & 0.0149 & 12.1 & 456 & \begin{tabular}{l|}
0.00625 \\
\end{tabular} & 0.00692 & 87.4 & 0.0177 & 42.3 & 256 & 0.391 & 0.400 & 9.04 & \begin{tabular}{l|l|}
0.413 \\
\end{tabular} & 3.51 & 103 \\
\hline & 0.0250 & 0.0320 & 11.7 & 0.0448 & 10.6 & 140 & 0.0250 & 0.0220 & 36.8 & 0.0749 & 0.539 & 341 & 1.56 & 1.53 & 7.00 & 1.54 & 7.9 & 101 \\
\hline & 0.100 & 0.0925 & 8.52 & 0.156 & 8.48 & 168 & 0.100 & 0.108 & 2.97 & 0.199 & 12.7 & 183 & 6.25 & 6.24 & 3.40 & 5.73 & 13.6 & 91.8 \\
\hline & 0.400 & 0.402 & 5.18 & 0.543 & 1.03 & 135 & 0.400 & 0.394 & 8.46 & 0.485 & 9.81 & 123 & 25.0 & 25.1 & 3.71 & 23.4 & 11.4 & 93.2 \\
\hline & 1.60 & 1.60 & 4.10 & 1.70 & 3.91 & 106 & 1.60 & 1.60 & 4.38 & 1.25 & 0.800 & 78.1 & 100 & 100.0 & 3.66 & 93.2 & 8.28 & 93.2 \\
\hline \multirow[t]{6}{*}{ APO } & 0 & & & 0.00000 & - & & 0 & 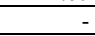 & & 0.00 & 0 & & 0 & & & $\begin{array}{ll}0.107 \\
\end{array}$ & 88.1 & \\
\hline & 0.00625 & 0.00342 & 51.3 & 0.0275 & 10.4 & 806 & 0.00625 & 0.00883 & 160 & 0.0154 & 21.3 & 175 & 0.391 & 0.453 & 15.83 & 0.511 & 8.18 & 113 \\
\hline & 0.0250 & 0.0332 & 29.9 & 0.114 & 60.3 & 344 & $\begin{array}{l}0.0250 \\
\end{array}$ & $\begin{array}{l}0.0219 \\
\end{array}$ & 75.1 & 0.0842 & 26.5 & 384 & 1.56 & 1.41 & 15.0 & 1.70 & 5.64 & 121 \\
\hline & 0.100 & 0.117 & 34.5 & 0.237 & 28.9 & 202 & 0.100 & 0.115 & 28.3 & 0.260 & 14.8 & 225 & 6.25 & 5.65 & 10.0 & 6.40 & 5.64 & 113 \\
\hline & 0.400 & 0.376 & 13.9 & 0.717 & 9.25 & 191 & 0.400 & 0.385 & 18.6 & 0.624 & 3.71 & 162 & 25.0 & 25.90 & 1.77 & 25.0 & 3.62 & 96.7 \\
\hline & 1.60 & 1.59 & 21.1 & 2.04 & 5.46 & 128 & 1.60 & 1.60 & 13.4 & 1.72 & 11.8 & 107 & 100 & 99.7 & 1.47 & 101 & 5.4 & 101 \\
\hline \multirow[t]{6}{*}{ AMPO } & & & & 0.00232 & 139 & & 0 & - & 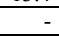 & 0 & 0 & & 0 & - & & 0.149 & 82.9 & \\
\hline & 0.00625 & 0.00144 & 114 & 0.0505 & 28.0 & 3517 & 0.00625 & 0.00810 & 173 & 0.0114 & 54.1 & 140 & 0.391 & 0.475 & 14.52 & 0.635 & \begin{tabular}{|l|}
10.9 \\
\end{tabular} & 134 \\
\hline & 0.0250 & 0.0431 & 39.3 & 0.146 & 54.5 & 340 & 0.0250 & 0.00808 & 172 & 0.121 & 29.7 & 1494 & 1.56 & 1.35 & 19.08 & 1.89 & $\begin{array}{l}7.63 \\
\end{array}$ & 140 \\
\hline & 0.100 & 0.124 & 21.7 & 0.279 & 30.9 & 225 & \begin{tabular}{l|l|}
0.100 \\
\end{tabular} & 0.143 & 59.6 & 0.332 & 22.1 & 232 & 6.25 & 5.49 & 13.3 & 6.95 & \begin{tabular}{l|l|}
8.32 \\
\end{tabular} & 127 \\
\hline & 0.400 & 0.362 & 18.3 & 0.832 & 14.1 & 230 & 0.400 & 0.357 & 44.5 & 0.806 & 3.91 & 225 & 25.0 & 26.10 & 1.92 & 26.5 & $\begin{array}{l}6.06 \\
\end{array}$ & 102 \\
\hline & 1.60 & 1.59 & $\begin{array}{ll}19.9 \\
\end{array}$ & 2.16 & 7.31 & 136 & $\begin{array}{l}1.60 \\
\end{array}$ & 1.60 & 16.2 & 2.05 & 7.90 & 128 & 100 & 99.7 & 0.31 & \begin{tabular}{|l|}
99.6 \\
\end{tabular} & 2.16 & 100 \\
\hline \multirow{6}{*}{ AAPO } & 0 & & & 0.00 & - & & 0 & & - & 0 & 0 & & 0 & 4 & - & \begin{tabular}{l|}
0.0768 \\
\end{tabular} & \begin{tabular}{|l|}
83.4 \\
\end{tabular} & - \\
\hline & 0.00625 & 0.00454 & 47.3 & 0.0128 & 4.59 & 281 & \begin{tabular}{l|}
0.00625 \\
\end{tabular} & 0.00536 & 68.9 & $\begin{array}{l}0.00731 \\
\end{array}$ & \begin{tabular}{|l|}
30.7 \\
\end{tabular} & 136 & \begin{tabular}{|l|}
0.391 \\
\end{tabular} & 0.417 & 7.63 & $\begin{array}{ll}0.497 \\
\end{array}$ & 15.0 & 119 \\
\hline & 0.0250 & 0.0295 & 15.2 & 0.0538 & 20.8 & 182 & $\begin{array}{r}0.0250 \\
\end{array}$ & 0.0261 & 6.89 & 0.0600 & 14.7 & 229 & 1.56 & 1.53 & 15.7 & 1.66 & \begin{tabular}{|l|}
8.43 \\
\end{tabular} & 109 \\
\hline & 0.100 & 0.114 & 20.2 & 0.149 & 21.2 & 131 & 0.100 & 0.114 & 6.56 & $\begin{array}{l}0.193 \\
\end{array}$ & 15.6 & 169 & 6.25 & 5.83 & 7.82 & 6.11 & 12.8 & 105 \\
\hline & 0.400 & 0.380 & 7.94 & 0.543 & 3.51 & 143 & 0.400 & 0.382 & 3.10 & 0.499 & 8.32 & 131 & 25.0 & 25.53 & 0.904 & 24.1 & 9.43 & 94.4 \\
\hline & 1.60 & 1.60 & 14.7 & 1.86 & 7.00 & 116 & 1.60 & 1.61 & 8.50 & 1.46 & 8.54 & 91.1 & 100 & 99.6 & 0.637 & 96.5 & $\begin{array}{l}8.06 \\
\end{array}$ & 96.9 \\
\hline \multirow[t]{6}{*}{ AAMPO } & 0 & & & 0.00 & & & 0 & & & 0 & 0 & & 0 & & & 0.106 & 0 & \\
\hline & 0.00625 & 0.00532 & 40.8 & 0.0113 & 30.1 & 212 & 0.00625 & 0.00794 & 98.3 & 0 & 0 & 0 & 0.391 & 0.424 & 13.8 & 0.514 & 7.34 & 121 \\
\hline & 0.0250 & 0.0279 & 13.5 & 0.0506 & 18.7 & 181 & 0.0250 & 0.0232 & 33.2 & 0.0432 & 30.3 & 186 & 1.56 & 1.53 & 22.8 & 1.74 & \begin{tabular}{|l|}
8.58 \\
\end{tabular} & 113 \\
\hline & 0.100 & 0.105 & 19.6 & 0.131 & 21.1 & 124 & $\begin{array}{l}0.100 \\
\end{array}$ & 0.115 & 14.5 & 0.180 & \begin{tabular}{ll|}
15.3 \\
\end{tabular} & 157 & 6.25 & $\begin{array}{r}5.62 \\
\end{array}$ & 11.9 & $\begin{array}{ll}6.18 \\
\end{array}$ & \begin{tabular}{|l|}
6.67 \\
\end{tabular} & 110 \\
\hline & 0.400 & 0.391 & 1.07 & 0.490 & 2.97 & 126 & 0.400 & 0.384 & 4.44 & 0.471 & 7.17 & 123 & 25.0 & \begin{tabular}{|l|}
25.83 \\
\end{tabular} & 0.59 & 24.6 & 3.06 & 95.1 \\
\hline & 1.60 & 1.60 & 12.9 & 1.73 & 11.3 & 108 & \begin{tabular}{ll|}
1.60 \\
\end{tabular} & 1.60 & 8.74 & 1.36 & 6.54 & 84.8 & 100 & 100 & 1.05 & \begin{tabular}{|l|}
94.8 \\
\end{tabular} & \begin{tabular}{|l|}
0.86 \\
\end{tabular} & 94.8 \\
\hline
\end{tabular}

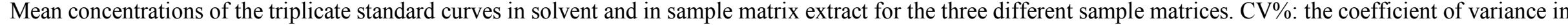

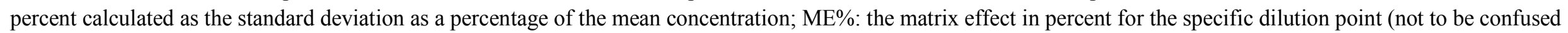

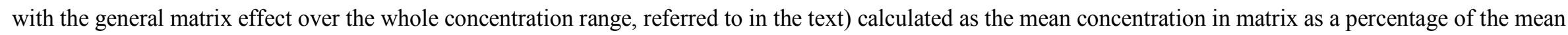
concentration in solvent. 
Supplementary Table 3. Recovery. During spiking and extraction of blank samples for recovery estimations all volumes were added by an automatic pipette of appropriate capacity. Due to the uncertainty of the pipettes every pipetted volume was immediately weighed. SD: Standard deviation. CV\%: Coefficient of variance.

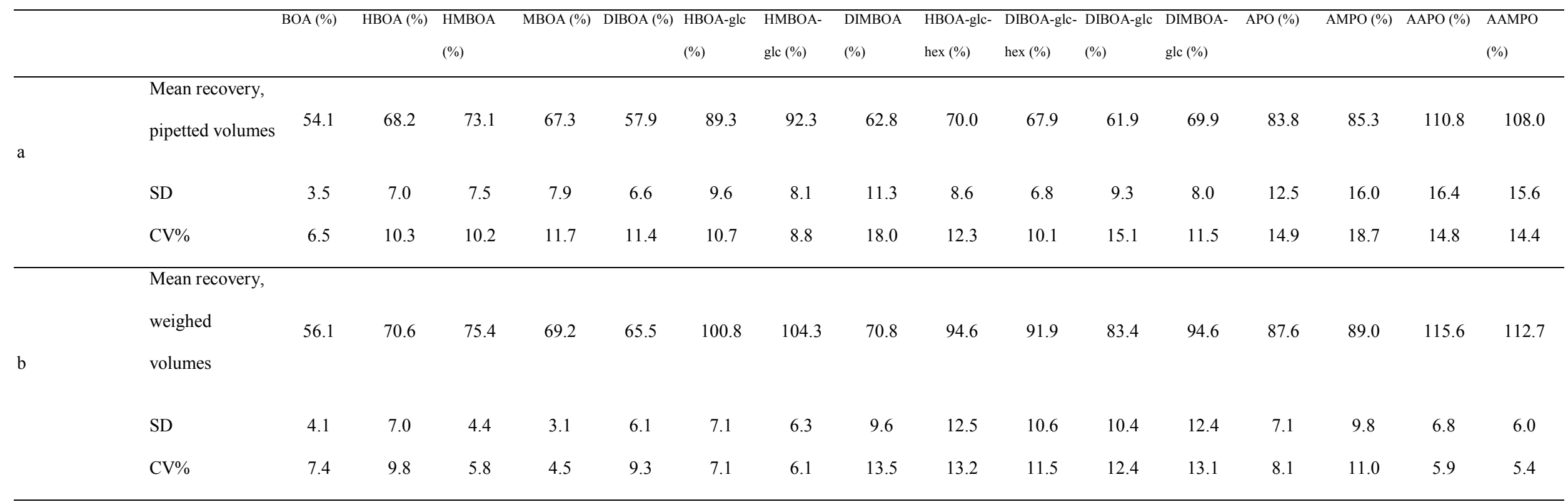

